\title{
ACOMPANHAMENTO PRÉ-NATAL PRECOCE E A INFLUÊNCIA NO DESFECHO DA GESTAÇÃO: ANÁLISE DO PERFIL EPIDEMIOLÓGICO DE GESTANTES EM UNIDADES DE SAÚDE DO MUNICÍPIO DE PALMAS-TO
}

\author{
EARLY PRENATAL FOLLOW-UP AND THE INFLUENCE ON THE \\ OUTCOME OF PREGNANCY: ANALYSIS OF THE EPIDEMIOLOGICAL \\ PROFILE OF PREGNANT WOMEN IN HEALTH UNITS IN THE \\ MUNICIPALITY OF PALMAS-TO
}

Brenda Caroline da Silveira Dias ${ }^{1}$, Palloma de Sá Antunes Bezerra², Gleyssi Couto de Souza Gomes ${ }^{1}$, Caroline Barros Figueira ${ }^{1}$, Manuela Bandeira da Silva Filha $^{1}$, Wilses de Sousa Tapajos Jacome ${ }^{3}$.

\section{ACESSO LIVRE}

Citação: Dias BCS, Bezerra PSA, Gomes GCS, Figueira $C B$, Filha MBS, Jacome WST (2021) Acompanhamento pré-natal precoce e a influência no desfecho da gestação: análise do perfil epidemiológico de gestantes em unidades de saúde do município de Palmas-TO. Revista de Patologia do Tocantins, 8(2).

Instituição: ${ }^{1}$ Acadêmico (a) do curso de Medicina da Universidade de Gurupi UNIRG, Gurupi, Tocantins, Brasil. ${ }^{2}$ Acadêmico (a) do curso de Medicina, Universidade Federal do Tocantins, Palmas, Tocantins, Brasil. ${ }^{3}$ Médica graduada pela Universidade Federal do Tocantins - UFT.

Autor correspondente: Palloma de Sá Antunes Bezerra. Universidade Federal do Tocantins - UFT. E-mail: pallomabezerra@hotmail.com. Endereço postal: Rua Major Dário Ferraz de Sá, 62, centro, Carnaubeira da Penha, Pernambuco, Brasil

Editor: Rosa A. C. G. Medicina, Universidade Federal do Tocantins, Brasil.

Publicado: 25 de julho de 2021.

Direitos Autorais: (c 2021 Dias et al. Este é um artigo de acesso aberto que permite o uso, a distribuição e a reprodução sem restrições em qualquer meio, desde que o autor original e a fonte sejam creditados.

Conflito de interesses: os autores declararam que não existem conflitos de interesses.

\begin{abstract}
RESUMO
Objetivos: Analisar o acompanhamento pré-natal precoce e sua influência no desfecho da gestação. Observar o perfil epidemiológico das gestantes em Palmas, Tocantins. Métodos: Estudo quantitativo e transversal realizado entre o período de janeiro de 2016 a janeiro de 2019 nos centros de saúde comunitária (CSC) das quadras 406 Norte e 210 sul em Palmas- Tocantins. Foram avaliadas 178 gestantes por meio de análise do prontuário eletrônico (E-SUS). As análises observacionais foram realizadas usando Microsoft Excel-2010. As variáveis descritas relacionadas a um pré-natal, foram: idade da paciente, raça, estado marital, escolaridade, renda, início do pré-natal, consultas ambulatoriais e visitas domiciliares. Resultados: o perfil epidemiológico encontrado no estudo foi de mulheres com idade média de 26 anos, as pacientes pardas totalizaram $54 \%$ das gestantes, o nível de escolaridade das gestantes participantes do estudo, cerca de $51 \%$ do total possuía nível de escolaridade de ensino básico completo (alfabetização), $77 \%$ das gestantes iniciaram o pré-natal no primeiro trimestre, cerca de $65 \%$ das pacientes tiveram sete ou mais consultas durante o pré-natal e foram encaminhadas ao serviço de pré-natal de alto risco $19 \%$ das gestantes participantes do estudo. Conclusão: 0 acompanhamento pré-natal de forma precoce contribui para uma gestação mais segura, além de possibilitar intervenções precoces e um tratamento adequado para mãe e feto.
\end{abstract}

PALAVRAS-CHAVES: Pré-natal; gestação; perfil epidemiológico; desfecho.

\begin{abstract}
Objectives: To analyze early prenatal follow-up and its influence on pregnancy outcome. Observe the epidemiological profile of pregnant women in Palmas, Tocantins. Methods: Quantitative and cross-sectional study carried out between January 2016 and January 2019 in community health centers (CSC) of the 406 north and 210 south blocks in Palmas- Tocantins. A total of 178 pregnant women were evaluated by analyzing the electronic medical records(E-SUS).

Observational analyses were performed using Microsoft Excel-2010. The variables described related to prenatal care were: age of the patient, race, marital status, education, income, beginning of prenatal care, outpatient consultations and home visits. Results: The epidemiological profile found in the study was of women with a mean age of 26 years, the brown patients totaled $54 \%$ of the pregnant women, the level of education of pregnant women participating in the study, about $51 \%$ of the total had a level of education of complete primary education (literacy), $77 \%$ of pregnant women started prenatal care in the first trimester, about $65 \%$ of patients had seven or more visits during prenatal care and $19 \%$ of the pregnant women participating in the study were referred to the high-risk prenatal service. Conclusion: Early prenatal follow-up contributes to a safer pregnancy, as well as enabling early interventions and appropriate treatment for mother and foetus.
\end{abstract}

KEYWORDS: Prenatal care; pregnancy; epidemiological profile; Outcome. 
avaliadas 178 gestantes por meio de análise do prontuário eletrônico (E-SUS). Esse estudo teve aprovação do Comitê de Ética em pesquisa com número CAAE: 14604119.6.0000.5553.

Ao longo do século $X X$, iniciou-se ações que visavam acompanhar a saúde da mãe e do seu embrião através de visitas domiciliares feitas por médicos, ações prioritárias para assistência à mulher, com enfoque nos aspectos da criança, gravidez e do parto, além de verificar a necessidade de internação hospitalar a depender do quadro materno. Com a redução da mortalidade materna e fetal principalmente devido à assistência pré-natal, este obteve uma importância e evolução contínua, onde foi associado exames e consultas periódicas durante todo o período gestacional. ${ }^{1}$

A organização das unidades de Atenção Básica de Saúde (ABS) é de grande valia para proporcionar o acesso e o acolhimento de todas as mulheres durante o ciclo gravídico-puerperal, possibilitando a realização de exames e consultas periódicas necessárias durante essa fase. Pode-se ainda, garantir quando necessário, ambulatórios especializados e hospitalares. ${ }^{2,3}$

A abordagem durante o período gestacional deve visar atividades de prevenção e promoção à saúde, o tratamento adequado e a cura das patologias e dos agravos. Ressalta-se, a relevância em conhecer o perfil socioeconômico no qual a paciente está inserida, as doenças e intercorrências diagnosticadas e seus manejos, os exames realizados, as condições de saúde da região, como também ter um conhecimento dos serviços de saúde oferecidos e se há profissionais e centros hospitalares especializados para o manejo adequado desse público alvo, objetivando uma gestação mais segura. ${ }^{4}$

Para muitas mulheres, a consulta pré-natal acaba sendo a única chance de analisar seu estado de saúde, sendo importante valorizar esse momento, como uma oportunidade para que os profissionais possam atuar integralmente na promoção e se necessário, recuperação da saúde. ${ }^{2}$ Cabe ainda ressaltar, a discrepância existente em relação ao número de consultas e da qualidade desiguais que há entre as raças, faixa etária, renda, entre outras variáveis de cada gestante.

Portanto, justifica-se a realização do presente estudo pela finalidade de analisar o desfecho da gestação de mulheres que fizeram o acompanhamento pré-natal e sua relação com o perfil epidemiológico; para se ter conhecimento dos benefícios trazidos e da seguridade para o binômio mãe-filho. Essa hipótese, se reafirmada, demonstra o valor que se tem ao realizar uma assistência pré-natal acertada. Por isso, objetivouse analisar o acompanhamento pré-natal precoce e sua influência no desfecho da gestação, além disso observar o perfil epidemiológico das gestantes na cidade de Palmas, Tocantins.

\section{METODOLOGIA}

Excel-2010, através de uma planilha contendo diversas variáveis.

Como critério de inclusão estão mulheres férteis, em idade reprodutiva, dos 16 aos 44 anos com média de 26 anos. Sendo critério de exclusão idades abaixo de 16 anos e acima de 44 anos. Outro critério de exclusão foram mulheres que não são assistidas nas localidades citadas e que não realizaram o prénatal completo.

Foram avaliadas 178 gestantes por meio de consultas, exames pré-natais, visitas domiciliares e a via de parto de escolha. As variáveis descritas foram relacionadas a um pré-natal fidedigno, tais como: idade da paciente, raça, estado marital, escolaridade, renda, início do pré-natal, consultas ambulatoriais e visitas domiciliares respectivamente do 10,20 e 3 ㅇ trimestre.

Os exames abrangeram além de dosagens laboratoriais, testes rápidos, ultrassonografias (USG), e fundamentalmente o exame físico constituído de altura de fundo uterino (AFU) e batimentos cardiofetais (BCF), todos realizados no 1으, 2으 e 3 ㅇ. Esse estudo, permite informar de maneira reprodutiva pacientes que concluíram o pré-natal de maneira fidedigna, isso é evidenciado pelo baixo porcentual de pacientes que foram encaminhadas ao alto risco.

\section{RESULTADOS E DISCUSSÕES}

O perfil epidemiológico encontrado no estudo foi de mulheres com idade média de 26 anos, o que corroborou com resultados encontrados em estudos semelhantes, realizados em outras regiões do país, como Anversa et al. (2012), no qual a idade média encontrada foi de 25 anos, e Tomasi et al. (2017), com 43,6\% entre $25-34$ anos.

As pacientes pardas totalizaram $54 \%$ das gestantes, dado que corrobora o perfil étnico/racial evidenciado na região Norte do país e que, portanto, torna necessária a análise da qualidade da assistência pré-natal prestada, pelo conhecido risco aumentado dessas pacientes receberem assistência inadequada à gestação e ao parto, resultantes de um processo de disparidade racial evidente. ${ }^{9}$

Com relação ao nível de escolaridade das gestantes participantes do estudo, cerca de $51 \%$ do total possuía nível de escolaridade de ensino básico completo (alfabetização). Segundo o Departamento de Informática do Sistema Único de Saúde (DATASUS), no ano de 2012, a proporção de gestantes que realizou seis ou mais consultas de pré-natal foi de $73 \%$, porém este percentual se mostrou menor em mulheres menos escolarizadas, mais jovens e com nível socioeconômico mais baixo, demonstrando maior tendência dessas pacientes à assistência de menor qualidade. ${ }^{8,11-13}$

Com relação ao trimestre de início da assistência pré-natal, $77 \%$

Trata-se de estudo quantitativo, observacional do tipo transversal realizado entre o período de janeiro de 2016 a janeiro de 2019 nos centros de saúde comunitária (CSC) das quadras 406 Norte e 210 Sul em Palmas- Tocantins. Foram das gestantes iniciaram o pré-natal no primeiro trimestre, diferindo dos resultados encontrados em estudo semelhante realizado na região do Sul do país, no ano de 2002, em que o percentual de gestantes, usuárias do Sistema Único de Saúde, 
que iniciaram o pré-natal até a décima quarta semana de gestação foi de apenas $34,7 \%^{14}$, dado que pode evidenciar melhora na qualidade da atenção à gestante nas últimas décadas. Outro estudo brasileiro demonstrou que o início precoce do acompanhamento pré-natal atinge apenas três quartos das gestantes, sendo menor naquelas pacientes mais jovens, de pele negra e residentes das regiões Norte e Nordeste do Brasil. 8,10

Cerca de $65 \%$ das pacientes tiveram sete ou mais consultas durante o pré-natal. Alguns estudos demonstraram que o nível de qualidade da assistência pré-natal está diretamente relacionado com o seu início precoce e a realização de seis consultas ou mais durante a gestação, dentre outros fatores ${ }^{5-7}$, apesar da divergência comum na literatura acerca do número médio de consultas, a Organização Mundial da Saúde (OMS), recomenda que sejam realizadas, pelo menos, seis consultas ao longo de todo o pré-natal, demonstrando que o maior percentual de gestantes participantes do estudo estão acima do parâmetro recomendado.

Além disso, foram encaminhadas ao serviço de pré-natal de alto risco $19 \%$ das gestantes participantes do estudo, percentual que poderia ter sido mais alto caso as pacientes não fossem submetidas a um pré-natal precoce e adequado de acordo com as recomendações do Ministério da Saúde.

\section{CONCLUSÕES}

O acompanhamento médico durante a gravidez busca garantir o bem-estar da mãe e do feto, possibilitando o acolhimento, assistência voltada ao aconselhamento para atender dúvidas e anseios maternos, realização de consultas e exames no tempo oportuno e intervenção sempre que constatada a necessidade. O pré-natal é fundamental para evolução de uma gestação segura visto que ele permite o diagnóstico precoce de patologias maternas e fetais, abreviando o início do tratamento e possibilitando conduta adequada. Essa assistência à gestante evitar maiores agravos e reduz a morbimortalidade, mudando o desfecho para ambos. Nesse contexto, foi observado que o perfil epidemiológico materno tem grande importância e influencia diretamente na busca pelo serviço, principalmente no que diz respeito à realização das consultas e exames preconizados no tempo adequado e ao decorrer de toda gestação.

A análise dos dados obtidos através da coleta realizada nas unidades de saúde supracitadas, mostra que $19 \%$ das pacientes avaliadas no período, foram classificadas como gestação de Alto Risco. Isso corresponde a um percentual importante de mulheres que são assistidas pela equipe de saúde de acordo com as necessidades do quadro clínico apresentado e consequentemente, maiores chances de melhora do prognóstico materno-fetal.

Ainda que o Sistema Único de Saúde (SUS) ofereça o acompanhamento de forma integral e gratuita, o nível de informação da gestante e o acesso aos serviços é decisivo para efetividade na prestação dessa assistência. Outro fator que pode ser considerado, porém, de difícil controle, é a disparidade no atendimento considerando as características socioeconômicas da paciente.
Com base na referência utilizada, ficou demonstrado que a efetivação do acompanhamento pré-natal é reduzida ao considerar as mães com idade mais baixa, pele negra e residentes nas regiões norte e nordeste o que permite considerar a integralidade do cuidado, a capacitação dos profissionais de saúde e educação e conscientização da população como fatores primordiais e que precedem a procura e a realização de um pré-natal de qualidade.

Ao considerarmos que $77 \%$ das gestantes iniciaram as consultas ainda no primeiro trimestre, percebe-se o aumento como um resultado positivo à assistência pré-natal nas unidades do município de Palmas-TO em estudo, no período cronológico especificado, na qual permitiu o acompanhamento adequado das gestações e ainda, intervir nos casos de risco possibilitando maiores chances de término da gestação favorável à mãe e seu filho.

Exemplifica-se, portanto, que o estudo epidemiológico das pacientes, permite conhecer e analisar características importantes ao início e andamento das consultas do pré-natal, levando em consideração ainda, o perfil socioeconômico da paciente e a possibilidade de interferir na busca pela Unidade de saúde ou ainda na forma como essa assistência é prestada. Entender este processo é crucial para que busquemos assistir a todas as gestantes de forma humanizada, com inclusão daquelas consideradas marginalizadas e tendo em vista as individualidades que cada uma pode apresentar, ofertando um serviço integral e que efetivamente atenda às necessidades maternas e fetais.

\section{REFERÊNCIAS BIBLIOGRÁFICAS}

1. 1- Cruz RSBLC, Caminha MFC, Batista Filho, M. Aspectos Históricos, Conceituais e Organizativos do Pré-natal. Rev. Brasileira de Ciências da Saúde. 2014; 18(1):87-94. Recife, PE.

2. 2- Brasil. Pré-natal e puerpério: atenção qualificada e humanizada: manual técnico. Brasília: Ministério da Saúde, 2006.

3. 3- American college of obstetricians and gynecologists. Clinical management guidelines for obstetriciangynecologists. ACOG Practice Bulletin. Obstet Gynecol 2003; 102(1):203-13.

4. 4- Brasil. Ministério da Saúde. Protocolos da Atenção Básica : Saúde das Mulheres / Ministério da Saúde, Instituto Sírio-Libanês de Ensino e Pesquisa - Brasília : Ministério da Saúde, 2016. 63-96 p.

5. 5- Ribeiro ERO, Guimarães AMDN, Bettiol $\mathrm{H}$, et al. Risk factors for inadequate prenatal care use in the metropolitan area of Aracaju. Northeast Brazil. BMC Pregnancy Childb. 2009; 9(31):1471-2393. PMid:19622174.

6. 6- César JA, Mendonza-Sassi RA, Gonzalez-Chica DA, et al. Características sociodemográficas e de assistência à gestação e ao parto no extremo sul do Brasil. Cad Saude Publica. 2011; 27(5):985-

94. http://dx.doi.org/10.1590/S0102311X2011000500016. PMid:21655849. 
7. 7- Anversa ETR, Bastos GAN, Nunes LN, Dal Pizzol TDS.

Qualidade do processo da assistência pré-natal: unidades básicas de saúde e unidades de Estratégia Saúde da Família em município no Sul do Brasil. Cad Saúde Pública. 2012; 28(4):789-800. http://dx.doi.org/10.1590/S0102311X2012000400018. PMid:22488324.

8. 8-Tomasi E, Fernandes PAA, Fischer T, et al. Qualidade da atenção pré-natal na rede básica de saúde do Brasil: indicadores e desigualdades sociais. Cadernos de Saúde Pública, 2017; 33(3): e00195815.

http://dx.doi.org/10.1590/0102-311x00195815.

9. 9- Leal MC; Gama SGN, Pereira APE, et al. A cor da dor: iniquidades raciais na atenção pré-natal e ao parto no brasil. Cadernos de Saúde Pública. 2017; 33(Suppl 1): e00078816. http://dx.doi.org/10.1590/0102$311 \times 00078816$.

10. 10- Silveira DS, Santos IS, Dias-da-Costa JS. Atenção prénatal na rede básica: uma avaliação da estrutura e do processo. Cad Saúde Pública. 2001; 17:131-9.

11. 11- Brasil. Indicadores e dados básicos: IDB Brasil [Internet]. 2020. Disponível em: http://tabnet.datasus.gov.br/cgi/idb2010/matriz.htm

12. 12- Rasia ICRB, Albernaz E. Atenção pré-natal na cidade de Pelotas, Rio Grande do Sul, Brasil. Rev Bras Saúde Matern Infant. 2008; 8:401-10.

13. 13-Viellas EF, Domingues RMSM, Dias MAB, Gama SGN, Theme Filha MM, Costa JV, et al. Assistência pré-natal no Brasil. Cad Saúde Pública. 2014; 30 Suppl 1:S85-100.

14. 14- Trevisan MR, De Lorenzi DRS, Araujo NM, Esber K. Perfil da Assistência Pré-Natal entre Usuárias do Sistema Único de Saúde em Caxias do Sul. Rev. Bras. Ginecol. Obstet. 2002; 24(5): 293-299. ISSN 18069339. https://doi.org/10.1590/S010072032002000500002. 